\title{
Unexpected Grain Size Reduction by Heating in Bulk Nanocrystalline FeAl
}

\author{
C. Gammer, ${ }^{* a, b}$, H.P. Karnthaler ${ }^{\mathrm{a}}$, C. Rentenberger ${ }^{\mathrm{a}}$ \\ ${ }^{a}$ University of Vienna, Physics of Nanostructured Materials, Boltzmanngasse 5, 1090 \\ Vienna, Austria \\ ${ }^{b}$ Present address: National Center for Electron Microscopy, Molecular Foundry, \\ Lawrence Berkeley National Laboratory, Berkeley, CA, USA
}

\begin{abstract}
The fact that heating can lead to a reduction of the grain size in an already grain-refined alloy is unexpected as the typical behavior would be grain growth. In contrast the grain size of bulk nanocrystalline intermetallic $\mathrm{FeAl}$ can be reduced further considerably by heating. The study is based on transmission electron microscopy investigations demonstrating an effect on the microstructural scale of fundamental importance in materials physics. Prior to heating the intermetallic alloy FeAl was deformed by severe plastic deformation, yielding a disordered nanocrystalline structure. By heating, the structure changes to an ordered nanocrystalline one with a 10 times reduced dislocation density and what is most striking with a grain size reduction by $50 \%$. This reduction occurs by converting small-angle grain boundaries into large-angle ones. In addition, the bulk annealing results were confirmed by in-situ heating in the transmission electron microscopy.
\end{abstract}

Key words: nanocrystalline materials, high-pressure torsion, recovery, ordering, transmission electron microscopy (TEM)

\section{Introduction}

Materials with a grain size less than $100 \mathrm{~nm}$ are called nanocrystalline $[1$, $2,3]$. The grains are defined by their large-angle grain boundaries having

\footnotetext{
* Corresponding author

Email address: christoph.gammer@univie.ac.at (C. Gammer)
} 
orientation differences $>15^{\circ}$ to the neighboring grains [4]. In a material made nanocrystalline by plastic deformation the grains contain a high density of dislocations that can arrange in small-angle boundaries $\left(<15^{\circ}\right)$ leading to a subgrain structure within the grains. It should be mentioned that the subgrain size corresponds to the size of the coherently scattering domains $\left(d_{C S D}\right)$ also called crystallite size as determined from diffraction experiments. Up till now it has been known that heating of a bulk nanocrystalline metal or alloy, leads to grain growth. Therefore, special measures must be undertaken even to keep the grain size constant during heating. This can be achieved by the addition of alloying elements leading to precipitations at the grain boundaries (e.g. oxides or carbides). The precipitates can act as grain growth blockers by hindering the movement of grain boundaries [5]. Recently, it was also suggested to suppress grain growth by using specific additional alloying elements that lead to a reduction of grain boundary energies $[6,7,8,9,10$, $11,12]$. It was reported that the suppression of grain growth correlated with appropriate changes in the concentration profiles of these alloying elements at the grain boundaries $[6,7]$.

In the case of $\mathrm{FeAl}$, it was shown that it is not only possible to avoid grain growth by heating, but a distinct reduction of the grain size $\left(d_{G}\right)$ is observed during heating [13]. To explain the unexpected reduction in grain size, methods of transmission electron microscopy (TEM) are applied to analyze carefully the changes of $d_{G}$ and $d_{C S D}$ caused by heating. By comparing them with the simultaneous change of the size of the chemically ordered domains $\left(d_{O D}\right)$, a model is worked out to explain the distinct reduction of the grain size as observed with heating. To get consistent data we deduced all values $\left(d_{G}, d_{C S D}, d_{O D}\right)$ from TEM results including both images and diffraction patterns.

\section{Experimental Procedure}

The iron rich $\mathrm{B} 2$ ordered Fe-45at.\% Al was made from high purity compounds and deformed by severe plastic deformation. For this the method of high pressure torsion (HPT) was used at room temperature (RT) achieving shear strains larger than $5,000 \%$ to reach a homogeneous nanocrystalline structure. The samples were heated to $130,170,220$ and $370^{\circ} \mathrm{C}$ at a heating rate of $20 \mathrm{~K} / \mathrm{min}$ followed by an immediate cooling process at a cooling rate of $20 \mathrm{~K} / \mathrm{min}$. TEM specimens were prepared from the periphery of the HPT samples by electropolishing. 
In addition to the information gained from TEM images, quantitative evaluations of the selected area electron diffraction (SAD) patterns were carried out. Recently we showed that this can be done successfully on a local scale in TEM by profile analysis based on SAD patterns (PASAD) [14]. Using X-ray methods a wide spread procedure to deduce $d_{C S D}$ of nanomaterials [15], is the modified Williamson-Hall plot $[16,17]$. When plotting the peak width of the diffraction peaks against the absolute value of their diffraction vectors, the size broadening and the strain broadening can be separated; also the contrast factors of the dislocations [18] can be taken into account to achieve a better agreement. The points of the plot lie on a straight line, where the slope of the line depends on the mean square strain and the intercept gives the size broadening. Electron diffraction profiles were deduced from the SAD patterns by azimuthal integration. The full width half maximum (FWHM) of the diffraction peaks were deduced by fitting pseudo-Voigt functions to all the peaks using the PASAD-tools [14]. From the size broadening $\left(\Delta g_{\text {size }}\right)$, $d_{C S D}$ is determined using the Scherrer equation: $d_{C S D}=0.886 \cdot\left(\Delta g_{\text {size }}\right)^{-1}$.

To analyze local orientation variations of the grains, we applied the method of reconstruction of diffraction patterns $[19,20]$. The reason is that standard methods to determine orientation variations as normally used in TEM and scanning electron microscopy are not applicable in the case of highly deformed, unrelaxed nanocrystalline materials. The method used is based on the reconstruction of diffraction patterns from a series of dark-field images [20]. A nanocrystal gives rise to distinct diffraction spots. When taking a dark-field image, this specific nanocrystal lights up only if the aperture

contains one of its diffraction spots. As a consequence by taking a series of dark-field images and scanning over the whole diffraction space it is possible to determine at which reciprocal positions the nanocrystal is in Bragg position and thus reconstruct the diffraction spots.

\section{Experimental Results}

\subsection{Small-angle grain boundaries and grain boundaries determined by diffrac- tion pattern reconstruction}

In Fig. 1 we illustrate the results of the TEM study of an as deformed sample. The TEM bright-field image (cf. Fig. 1a) shows strong contrast variations indicating that the sample is nanocrystalline but it is not possible to determine the grain boundaries. In this context, it should be pointed out that the contrast features of bulk nanocrystalline materials must be interpreted 
with special care since strong effects of moiré contrast appear frequently because of the overlapping nanograins [21]. Therefore the grain boundaries and the small-angle grain boundaries indicated by bold and dashed lines, respectively, in Fig. 1a had to be deduced from the evaluation of a series of dark-field images. The corresponding SAD pattern (cf. Fig. 1b) shows diffraction rings indicating that the grain orientations are about randomly distributed; superlattice reflections (e.g. $\{100\}$ ) are hardly observed as the severely deformed sample has a strongly reduced LRO [22]. Fig. 1c shows as an example two overlapping dark-field images (in red and green) indicating regions of different orientations that occur within a grain. The corresponding positions of the SA aperture are shown in Fig. 1b. Only crystals that are near Bragg-position for the given aperture position light up. In order to determine the orientation differences of neighboring regions, a reconstruction of their diffraction patterns was carried out. Therefore, more than 450 dark-field images were recorded, scanning along the $\{110\},\{200\}$ and $\{211\}$ diffraction rings. For each dark-field image we evaluated the mean intensity in the selected region to find out the corresponding intensity in the diffraction pattern. As the positions of the aperture along the diffraction rings were heavily oversampled, it is possible to deconvolve for the size of the aperture, thus obtaining the position of the diffraction spots with a high precision (in the present case around $\left.\pm 2^{\circ}\right)$. Fig. $1 d$ represents the reconstructed diffraction patterns from the regions encircled with red and green dashed lines in Fig. 1a. It leads to the result that their orientation difference is only $4 \pm 2^{\circ}$. Therefore, the two regions are separated by a small-angle grain boundary. The diffraction patterns correspond to an orientation close to [111]. (The intensities of the diffraction spots are indicated by their size.) In the same way we measured the tilt between neighboring subgrain regions revealing the presence of other small-angle grain boundaries (cf. Fig. 1a). In addition to the presented grain several other grains were studied showing also small-angle grain boundaries within the grains. Furthermore, the grain size analysis of grains with large-angle grain boundaries $\left(>15^{\circ}\right)$ was performed from series of dark-field TEM images, yielding a volume weighted mean grain-size diameter of $100 \pm 15 \mathrm{~nm}$ (this corresponds to a mean grain size of about $80 \mathrm{~nm}$ ).

Fig. 2 shows the TEM study of HPT deformed FeAl heated up to $370^{\circ} \mathrm{C}$. The bright-field image of Fig. 2a is used to sketch the grain boundaries that had to be deduced from a series of dark-field images as it is not possible to gain the correct information in the bright-field image. Fig. $2 b$ shows the corresponding SAD pattern that reveals the occurrence of superlattice reflec- 
tions (e.g. $\{100\}$-ring) indicating that long range order was restored during heating of the sample. In Fig. 2c the overprinting of two dark-field images is shown. To ensure that the regions lighting up in red and green (corresponding to the positions of the SA aperture indicated in Fig. 2b) represent grains, it is necessary to prove that they are separated by large-angle grain boundaries. Therefore we measure the orientation differences by reconstructing diffraction patterns. More than 450 dark-field images were recorded, scanning along the $\{110\},\{200\}$ and $\{211\}$ diffraction rings. Fig. 2d shows as an example the reconstructed diffraction patterns corresponding to the two grains imaged in Fig. 2c. The diffraction patterns have an orientation near [101], but they are rotated against each other by $20 \pm 2^{\circ}$, indicating a largeangle grain boundary. An other large-angle grain boundary $\left(24 \pm 2^{\circ}\right)$ was analyzed in Fig. 2. We also studied several regions of the sample giving similar results yielding a mean grain-size diameter of $49 \pm 5 \mathrm{~nm}$.

\subsection{Comparison of grain size with coherently scattering domain size and ordered domain size as function of temperature}

In addition to the unexpected result of the grain size it is of interest to study the CSD size. We determined the volume weighted mean CSD size for the as-deformed sample and for the samples heated up to different temperatures from a modified Williamson Hall plot taking into account fundamental reflections only. Furthermore we deduced the volume weighted mean ordered domain size $\left(d_{O D}\right)$ using only superlattice reflections [22]. It should be noted that from the modified Williamson Hall plot dislocation densities can also be obtained showing that the dislocation density decreases by a factor of $\sim 10$ after heating the sample up to $370^{\circ} \mathrm{C}$. This decrease of the dislocation density agrees well with the fact that the contrast of the grains is much more homogeneous in Fig. 2c than that in Fig. 1c.

Fig. 3 shows the three determined structural data as a function of temperature: the grain size, the CSD size and the size of the ordered domains. In this context we want to point out: (i) The value of the grain size is about $100 \mathrm{~nm}$ at RT, it stays constant up to $220^{\circ} \mathrm{C}$ and drops by a factor of $\sim 0.5$ when the sample is heated up to $370^{\circ} \mathrm{C}$. (ii) In contrast, the value of the CSD size is much smaller $(\sim 25 \mathrm{~nm})$ between RT and $220^{\circ} \mathrm{C}$ and increases to $\sim 41 \mathrm{~nm}$ at $370^{\circ} \mathrm{C}$. (iii) The ordered domains are nanosized after deformation at RT and grow by heating. This confirms that re-ordering is linked to the growth of ordered domains [22]. Immediately after the deformation the ordered domains are as small as $2 \pm 1 \mathrm{~nm}$; they grow to $10 \pm 3 \mathrm{~nm}$ at $220^{\circ} \mathrm{C}$ 
and reach the size of the grains being coincident with them at $370{ }^{\circ} \mathrm{C}$.

\subsection{Grain size reduction observed by in-situ heating in the TEM}

The results on the grain size given in the paper are referring to specimens heated up and cooled down in the bulk. This means the thermal treatment was done before the specimens were thinned to make electron transparent foils. This has the advantage that thin foil effects and oxidation of the foils in the TEM at elevated temperatures can be excluded. Still, we considered it worth to carry out an in-situ experiment in the TEM. In this case the thin foil is heated up in the TEM and images of a selected region are recorded simultaneously. Figure 4 shows bright field images taken from the same region at different temperatures showing a transformation of subgrain boundaries into large-angle grain boundaries. In this case a reduction of the grain size of more than $50 \%$ is yielded. Figure 5 shows dark field images taken using a fundamental and the corresponding superlattice reflection. The superlattice reflection shows the coarsening of the ordered domains while the fundamental reflection shows the transformation of a region of similar orientation into two grains.

\section{Discussion}

\subsection{Model to explain the grain size reduction}

In Fig. 6 the evolution of the grain size with temperature is schematically shown. Based on the careful TEM analysis leading to the quantitative results compiled in Fig. 3 we can draw a consistent picture to explain the unexpected grain size reduction occurring by heating. At the beginning of the deformation the structure of the specimen is long range ordered, therefore the glide dislocations are superlattice dislocations consisting of coupled unit dislocations separated by an antiphase boundary (APB) fault ribbon. Severe plastic deformation reduces the long range order as it is frequently observed in intermetallics $[23,24]$. Therefore at 5,000 \% of deformation a large fraction of uncoupled unit dislocations are expected to be present. The TEM study of severely deformed FeAl yielded that the reduced order is different from the statistical disorder that occurs near an order disorder temperature in other alloys [22]. During severe plastic deformation the frequent cutting of the deformation induced APB faults by the glide dislocations leads to very small ordered domains $\left(d_{O D} \sim 2 \mathrm{~nm}\right)$ as shown in Fig. 3 [25]. Fig. 6a illus-

trates schematically the structure prior to heating: One grain contains four 
subgrains (the ratio corresponds about to the result given in Fig. 3) that are separated by small-angle grain boundaries. The small-angle grain boundaries are formed by a local accumulation of dislocations. The subgrains contain small ordered domains $\left(d_{O D} \sim 0.1 d_{C S D}\right.$, cf. Fig. 3 ) bounded by APB (gray lines) and also a large density of dislocations.

Fig. $6 \mathrm{~b}$ and $\mathrm{c}$ shows the situation during heating. Heating leads to a strong growth of the ordered domains that is especially pronounced between 170 and $370{ }^{\circ} \mathrm{C}$ (cf. Fig. 3). Since this temperature range agrees with that of the pronounced exothermic peak observed in differential scanning calorimetry curves it is concluded that after heating to $370{ }^{\circ} \mathrm{C}$ the chemical LRO is restored [13]. The growth of the ordered domains causes a movement of the APB; this leads to the interaction with unit dislocations that are for topological regions connected to APB faults. As a consequence the unit dislocations are moved by the APB bounding the growing ordered domains (only the few superlattice dislocations remain as they do not disturb the LRO). The rearrangement of the dislocations leads to the result that some of the small-angle boundaries turn into large-angle boundaries whereas other small-angle boundaries are dissolved and also the dislocation density inside the grains is reduced by a factor of about ten. In contrast, the rearrangement of dislocations by annealing as reported in a pure metal after severe plastic deformation does not lead to a grain size reduction [27].

In accordance with the experimental finding Fig. 6c illustrates the resulting structure after heating up to $370^{\circ} \mathrm{C}$ : The grain size, the size of the the ordered domains and the size of the coherently scattering domains reach the same value $\left(d_{G}=d_{O D}=d_{C S D}\right)$. The dislocation incorporated in the smallangle grain boundaries have converted them into large-angle grain boundaries. Therefore, we propose that the reason for the reduction of the grain size is the interaction of the glide dislocation with the ordered domains growing during heating up. In the present case the driving force is caused by the phase transformation from the disordered structure to the ordered one.

The experimental results lead to the conclusion that for the grain size reduction by heating the necessary prerequisite is: a high density of both, dislocations and small angle grain boundaries inside the grain as caused by severe plastic deformation and a driving force moving the dislocations to the small angle boundaries. In addition, the grain size reduction must occur at a temperature below the one at which dislocation annihilation and grain growth occurs. 
4.2. Relation between the value of the grain size and the value of the coherently scattering domain size

In the present paper the values of the grain size $\left(d_{G}\right)$, the coherently scattering domain size $\left(d_{C S D}\right)$ and the ordered domain size $\left(d_{O D}\right)$ are compared with each other.The present results clarify the frequently arising discrepancy between the values of $d_{G}$ and $d_{C S D}$ in deformed materials [26]. It was thought that this discrepancy might be influenced by the method used to achieve the values since $d_{G}$ and $d_{C S D}$ were deduced from real space and reciprocal space data by TEM and X-ray methods, respectively. In the present study we determine all data in the TEM from identical regions of the same specimen. We reach a very good agreement between the data deduced from real space (TEM images) and reciprocal space (TEM diffraction patterns). Finally, we show that for the values of $d_{C S D}$ and $d_{G}$ the relation holds $d_{C S D} \leq d_{G}$ (as $d_{C S D}$ corresponds to the subgrain size). In the present case $d_{C S D}<d_{G}$ by a factor of 5 in the severely deformed material whereas in the relaxed structures $d_{C S D}=d_{G}$.

\section{Summary and Conclusions}

1. We show that careful TEM investigations are able to elucidate the unexpected grain reduction by heating occurring in bulk nanocrystalline FeAl. It is important to note that in addition to the specimens heated up in bulk and studied post mortem in the TEM also an experiment with heating the specimens in-situ in the TEM was carried out yielding a very good agreement.

2. The results are based on the evaluation of both, TEM images and TEM diffraction patterns to determine the three parameters: grain size $\left(d_{G}\right)$, the size of the coherently scattering domains $\left(d_{C S D}\right)$ and the size of the chemically ordered domains $\left(d_{O D}\right)$ in specimens heated up to various temperatures and cooled down to RT (both at the same rate of $20 \mathrm{~K} / \mathrm{min})$. Prior to heating the specimens were severely deformed by HPT $(>\sim 5000 \%)$ yielding a disordered nanocrystalline structure $\left(d_{G} \sim 100 \mathrm{~nm}\right)$ with $d_{C S D} \sim 0.2 d_{G}$ and $d_{O D} \sim 0.1 d_{C S D} \sim 0.02 d_{G}$.

3. By heating up to $370^{\circ} \mathrm{C}$ all three parameters $\left(\mathrm{dg}, d_{C S D}\right.$ and $\left.d_{O D}\right)$ reach the same value of about $45 \mathrm{~nm}$ and the structure changes to an ordered nanocrystalline structure with a reduced dislocation density (by a factor $\sim 10)$. This structure shows the unexpected result of a grain size reduction by heating (factor $\sim 0.5$ ). 
4. By making use of all the data a model is worked out describing how the grain size reduction can occur by converting small angle boundaries into large angle boundaries.

5. Finally, the nanostructures achieved by heating exhibit increased properties: e.g. hardness [13] and promising potential applications as shielding materials in fusion reactors as they facilitate enhanced irradiation resistance [28]. The present study is focused on FeAl. The method described may also apply to other ductile intermetallics that disorder and can be made nanocrystalline by severe plastic deformation. It has been predicted that a large number of ductile intermetallics exist [29]. Therefore, this result furnishes research avenues of both fundamental science and technological applications.

\section{Acknowledgments}

This work was supported by the Austrian Science Fund (FWF):[S10403, P22440] and the IC "Experimental Materials Science - Nanostructured Materials" of the University of Vienna. Work at the Molecular Foundry was supported by the Office of Science, Office of Basic Energy Sciences, of the U.S. Department of Energy under Contract No. DE-AC02-05CH11231.

\section{References}

[1] K. Lu, L. Lu, S. Suresh. Strengthening materials by engineering coherent internal boundaries at the nanoscale. Science 324 (2009) 349.

[2] Y. Wang, M. Chen, F. Zhou, E. Ma. High tensile ductility in a nanostructured metal. Nature 419 (2002) 912.

[3] R. Z. Valiev. Nanostructuring of metals by severe plastic deformation for advanced properties. Nature Materials 8 (2004) 511.

[4] F. J. Humphreys. Reconstruction of grains and subgrains from electron backscatter diffraction maps. Journal of Microscopy, 213 (2014) 247.

[5] K. Boylan, D. Ostrander, U. Erb, G. Palumbo, K.T. Aust. An in-situ tem study of the thermal stability of nanocrystalline Ni-P. Scripta Metallurgica et Materialia 25 (1991) 2711. 
[6] T. Chookajorn, H.A. Murdoch, C.A. Schuh. Design of stable nanocrystalline alloys. Science 337 (2012) 951.

[7] R. Kirchheim. Grain coarsening inhibited by solute segregation. Acta Materialia 50 (2002) 413.

[8] R. Kirchheim. Reducing grain boundary, dislocation line and vacancy formation energies by solute segregation: II. Experimental evidence and consequences. Acta Materialia 55 (2007) 5139.

[9] B. Faerber, E. Cadel, A. Menand, G. Schmitz, R. Kirchheim. Phosphorus segregation in nanocrystalline $\mathrm{Ni}-3.6$ at.\% $\mathrm{P}$ alloy investigated with the tomographic atom probe (TAP). Acta Materialia 48 (2000) 789.

[10] C. Krill, H. Ehrhardt, and R. Birringer. Thermodynamic stabilization of nanocrystallinity. Zeitschrift für Metallkunde, 96 (2005) 1134.

[11] R. Trelewicz, C.A. Schuh. Grain boundary segregation and thermodynamically stable binary nanocrystalline alloys. Phys. Rev. B 79 (2009) 094112.

[12] M.A. Atwater, R.O. Scattergood, and C.C. Koch. The stabilization of nanocrystalline copper by zirconium. Materials Science and Engineering: A 559 (2013) 250.

[13] C. Mangler, C. Gammer, H. P. Karnthaler, C. Rentenberger. Structural modifications during annealing of bulk nanocrystalline FeAl produced by high pressure torsion. Acta Mater. 58 (2010) 5631.

[14] C. Gammer, C. Mangler, C. Rentenberger, H.P. Karnthaler. Quantitative local profile analysis of nanomaterials by electron diffraction. Scripta Mater. 63 (2010) 312.

[15] E. Schafler, M. Zehetbauer. Characterization of nanostructured materials by X-ray Line Profile Analysis. Rev. Adv. Mater. Sci. 10 (2005) 28 .

[16] G.K. Williamson, W.H. Hall. X-ray line broadening from filed aluminium and wolfram. Acta Metall. 1 (1953) 22. 
[17] T. Ungar, A. Borbely. The effect of dislocation contrast on X-ray line broadening: A new approach to line profile analysis. Appl. Phys. Lett. 69 (1996) 3173.

[18] T. Ungar, I. Dragomir, A. Revesz, J. Appl. Crystallogr. 32 (1999) 992.

[19] H. H. Liu, S. Schmidt, H. F. Poulsen, A. Godfrey, Z. Q. Liu, J. A. Sharon, $\mathrm{X}$. Huang Three-Dimensional Orientation Mapping in the Transmission Electron Microscope. Science 332 (2011) 833.

[20] D.J. Dingley. Orientation Imaging Microscopy for the Transmission Electron Microscope. Microchim. Acta 155 (2006) 19.

[21] C. Rentenberger, T. Waitz, H.P. Karnthaler. HRTEM analysis of nanostructured alloys processed by severe plastic deformation) Scripta Mater. 51 (2004) 789 .

[22] C. Gammer, C. Mangler, H.P. Karnthaler, C. Rentenberger. Growth of nanosized ordered domains in intermetallic $\mathrm{FeAl}$ made nanocrystalline by severe plastic deformation. Scri. Mater. 65 (2011) 57.

[23] D. Geist, C. Gammer, C. Mangler, C. Rentenberger, and H. P. Karnthaler. Electron Microscopy of severely deformed L12 intermetallics. Phil. Mag. 90 (2010) 4635.

[24] C. Rentenberger, H.P. Karnthaler. Extensive disordering in long-rangeordered $\mathrm{Cu} 3 \mathrm{Au}$ induced by severe plastic deformation studied by transmission electron microscopy.Acta Mater 56 (2008) 2526.

[25] C. Mangler, C. Gammer, K. Hiebl, H.P. Karnthale, C. Rentenberger. Thermally induced transition from a ferromagnetic to a paramagnetic state in nanocrystalline $\mathrm{FeAl}$ processed by high-pressure torsion. Journal of Alloys and Compounds 509 Suppl.1 (2011) 389

[26] J. Gubicza, N.Q. Chinh , Z. Horita, T.G. Langdon. Effect of Mg addition on microstructure and mechanical properties of aluminum. Materials Science and Engineering A 387-389 (2004) 55-59

[27] X. Huang, N. Hansen, N. Tsuji. Hardening by Annealing and Softening by Deformation in Nanostructured Metals. Science 312 (2006) 249. 
[28] A. Kilmametov, A. Balogh, M. Ghafari, C. Gammer, C. Mangler, C. Rentenberger, R. Valiev, H. Hahn. Radiation effects in bulk nanocrystalline FeAl alloy. Radiat. Eff. Defect. S. 167 (2012) 631.

[29] K. Gschneidner, A. Russel, A. Pecharsky, J. Morris, Z. Zhang, T. Lograsso, D. Hsu, C.H. Lo, Y. Ye, A. Slager, D. Kesse. A family of ductile intermetallic compounds. Nature Materials, 9 (2003) 587. 

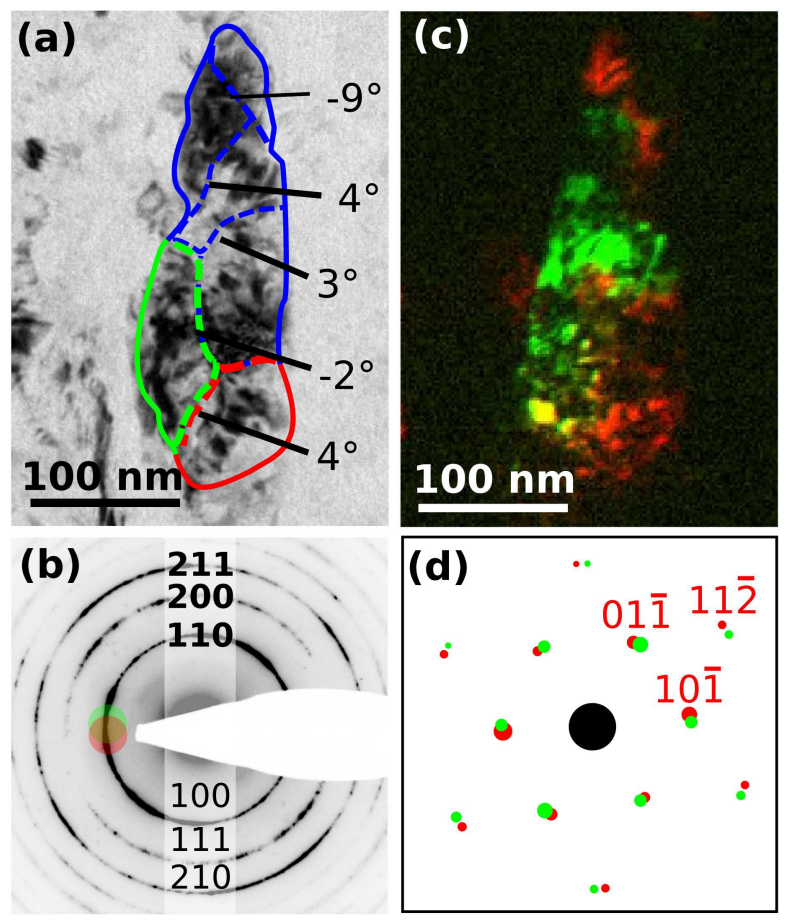

Figure 1: Nanocrystalline FeAl, as deformed by HPT at RT. (a) The subgrains within the grain are sketched on a bright-field image; the boundaries and their misorientations are determined from a series of dark-field images as explained in the text. (b) SA diffraction pattern; the intensity of superlattice reflections (e.g. $\{100\})$ is very low. (c) Overlapping dark-field images indicating different orientations within a grain. Red and green regions correspond to the positions of the SA aperture indicated in (b). (d) Reconstructed diffraction patterns of the red and green regions (encircled in (a)) leading to an orientation difference of $4 \pm 2^{\circ}$ of a small-angle grain boundary. 


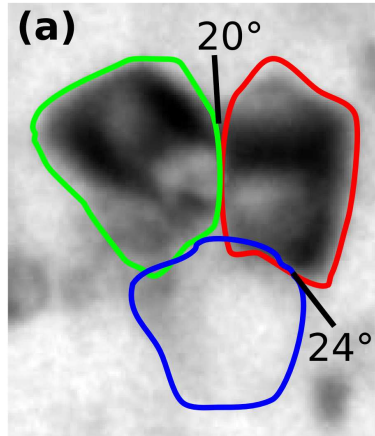

$30 \mathrm{~nm}$

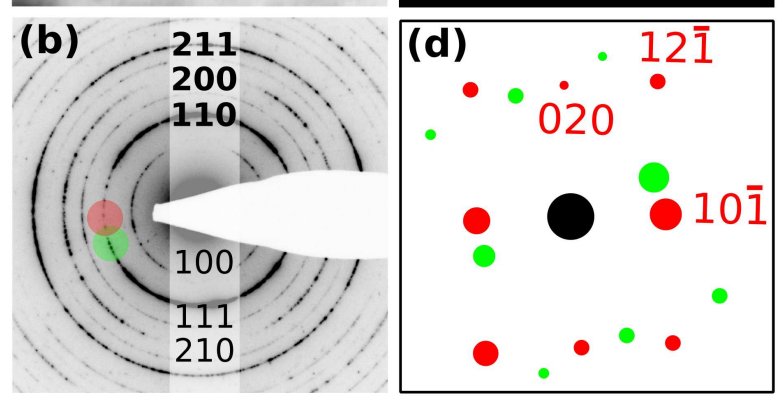

Figure 2: Nanocrystalline FeAl deformed by HPT and heated up to $370{ }^{\circ} \mathrm{C}$ and cooled down to RT at a rate of $20 \mathrm{~K} / \mathrm{min}$. (a) The bright-field image is used to sketch the grain boundaries and indicate the misorientations as deduced from a series of dark-field images. (b) SA diffraction pattern showing rings of both fundamental and superlattice reflections. (c) Overprinting of two dark-field images (in red and green) indicating two grains. The positions of the SA aperture used for the two images are given in (b). (d) Reconstructed diffraction patterns of the two grains (in red and green) yielding a largeangle grain boundary with an orientation difference of $20 \pm 2^{\circ}$. 


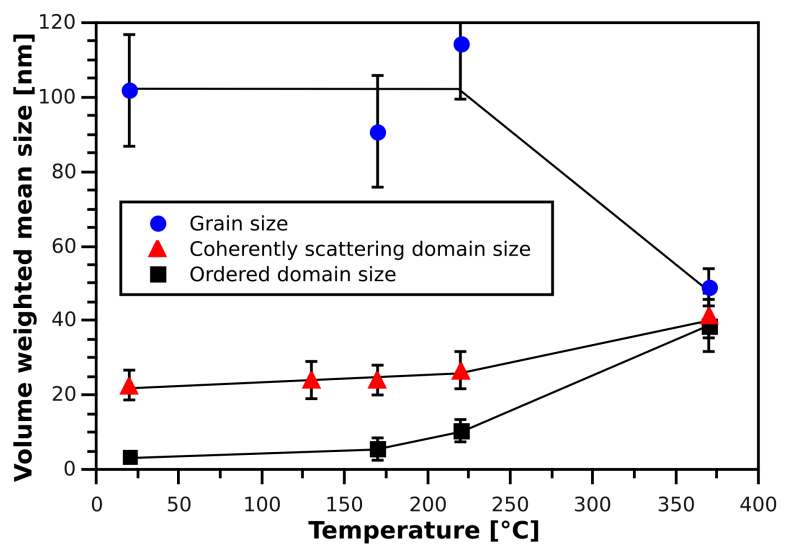

Figure 3: Variation of the mean coherently scattering domain size, the chemically ordered domain size and the grain size in FeAl deformed by HPT and heated up to different temperatures and cooled down to RT $(20 \mathrm{~K} / \mathrm{min})$. Volume weighted equivalent sphere diameters are used in all three cases. (The values of the ordered domain size are taken from $[22]$. 
(a)
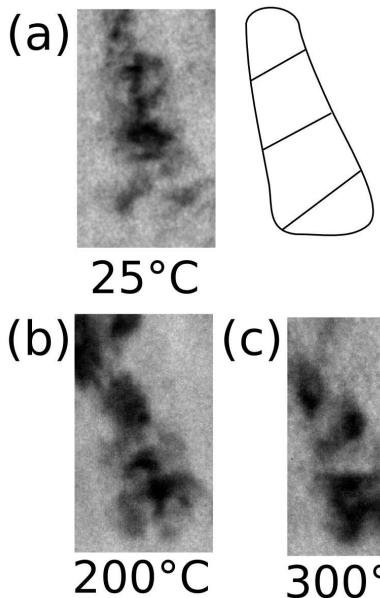

(c)

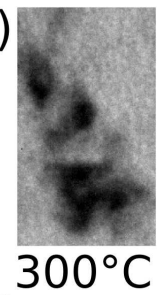

(d)
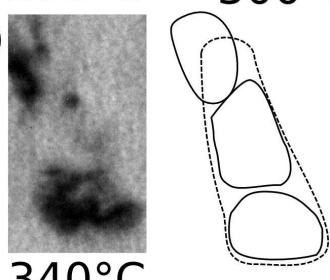

$50 \mathrm{~nm}$

Figure 4: In-situ heating observations in the TEM based on bright field images showing the reduction of the grain size. (a) Bright field image of a grain showing several subgrain boundaries as indicated in the sketch. (b and c) During heating the contrasts in the grain are reduced and the subgrain boundaries become sharper. (d) After heating to $340^{\circ} \mathrm{C}$ the grain transformed into 3 grains (cf. sketch) separated by large-angle grain boundaries. 


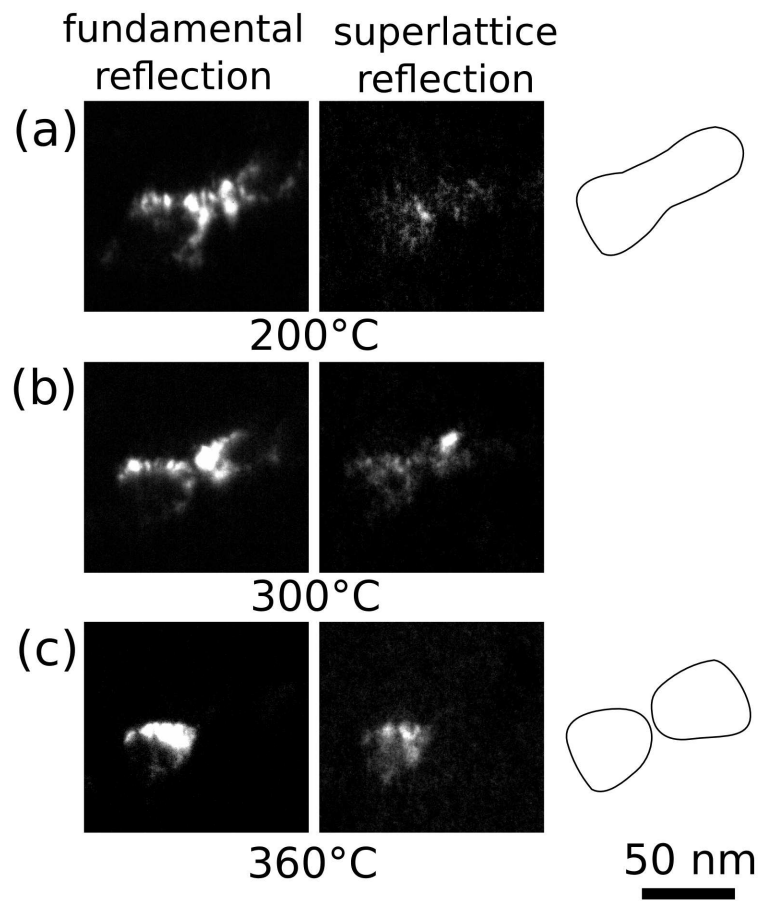

Figure 5: Dark-field images of in-situ heating observations. (a) The fundamental reflection shows a region containing several subgrain boundaries. The superlattice reflection shows small ordered domains $(\sim 2 \mathrm{~nm})$ within the region of the whole grain (as indicated in the sketch). (b) Heating leads to a coarsening of the small ordered domains. (c) After heating to $360^{\circ} \mathrm{C}$ the initial grain transformed into 2 grains (cf. sketch) separated by a largeangle grain boundary. As deduced from the image taken with a superlattice reflection the domain size corresponds to the grain size. 

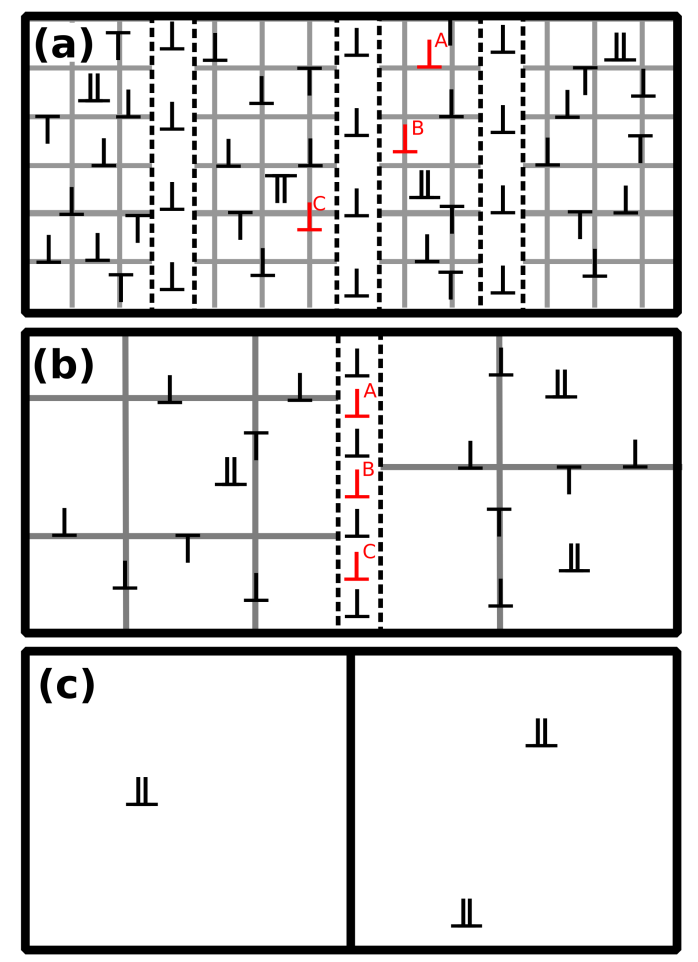

Figure 6: Sketch of the process proposed to explain the grain size reduction as based on the data of Fig. 3. (a) A grain bounded by a large-angle grain boundary (bold line) contains small-angle grain boundaries (dashed), APB faults (grey lines) and dislocations $(\perp)$ ). (b) When the specimen is heated up the growing ordered domains move the dislocations being connected by APB faults to the domain walls. (c) When heated up to $370^{\circ} \mathrm{C}$ the grain size, the CSD size and the size of the ordered domains reach the same value since the process described in (b) can transform small-angle boundaries into large-angle boundaries. The density of small-angle boundaries and that of dislocations is reduced, mainly superlattice dislocations $(\perp \perp)$ are remaining inside the grains in (c). 